\title{
Reactivity of commercially available monoclonal antibodies to human CD antigens with peripheral blood leucocytes of dromedary camels (Camelus dromedarius)
}

\author{
Jamal Hussen ${ }^{1, *}$, Turke Shawaf ${ }^{2}$, Abdulkareem Imran Al-herz ${ }^{3}$, Hussain R. Alturaifi ${ }^{3}$ and Ahmed M. Alluwaimi ${ }^{1}$ \\ ${ }^{I}$ Department of Microbiology and Parasitology, College of Veterinary Medicine, King Faisal University, Al Ahsaa, \\ Saudi Arabia \\ ${ }^{2}$ Department of Clinical Studies, College of Veterinary Medicine, King Faisal University, Al Ahsaa, Saudi Arabia \\ ${ }^{3}$ Immunology Unit, Diagnostic Laboratory and Blood Bank, King Fahad Hospital Hufof, Al Ahsaa, Saudi Arabia
}

\begin{abstract}
Monoclonal antibodies (mAbs) to cell surface molecules have been proven as a key tool for phenotypic and functional characterization of the cellular immune response. One of the major difficulties in studying camel cellular immunity consists in the lack of mAbs that dtect their leukocyte differentiation antigens. In the present study two-parameter flow cytometry was used to screen existing commercially available mAbs to human leukocyte antigens and major histocompatibility molecules (MHC) for their reactivity with camel leukocytes. The comparison of patterns of reactivity obtained after labelling human and camel leukocytes have shown that mAbs specific to human cluster of differentiation (CD) 18, CD11a, CD11b and CD14 are predicted to be cross-reactive with homologous camel antigens. Keywords: Antibodies, Cross-reactivity, Dromedary camel, Flow cytometry.
\end{abstract}

\section{Introduction}

The immune system consists of a complex network of cellular and non-cellular components, which interact with each other to protect the animal against invading pathogens like bacteria, fungi, parasites and viruses. In comparison to several other veterinary species like cattle (Hussen et al., 2013; Duvel et al., 2014), pigs (Gerner et al., 2015), sheep (Hopkins et al., 1993) and horses (Lunn et al., 1998), the immune system of camels remains to a great extent poorly studied. Although considerable progress has been made in the characterization of camel immunoglobulins (HamersCasterman et al., 1993; Muyldermans, 2013), few data are available on the cellular immunity of camels in health or disease (Zidan et al., 2000a,b; Al-Mohammed Salem et al., 2012).

Monoclonal antibodies (mAbs) to leukocyte antigens are highly important tools for phenotypic and functional analysis of cellular immunology. The lack of mAbs that define camel immune cells represents one of the major difficulties in studying the camel cellular immune response.

As the production of mAbs is very costly, attempts are made to study the cross-reactivity of commercially available mAbs to leukocyte antigens of one species with leukocytes of other species.

Comparative studies with $\mathrm{mAb}$ to leukocyte antigens of ruminants, swine, horses, and dogs have shown that the pattern of expression of many molecules is conserved cross species (Davis et al., 1995). These findings suggests that it would be useful to use comparative studies for the identification of mAbs that recognize conserved epitopes on leukocyte differentiation antigens in poorly studied species.

The identification of cross-reactive mAbs could reduce the need to develop reagents for some important molecules and would provide an opportunity to compare the immune systems of camel with that of other species.

The objective of the present study was to screen existing $\mathrm{mAbs}$ to human leukocyte antigens and major histocompatibility molecules (MHC) for their reactivity with camel leukocytes. This would help in identifying mAbs that could be used to study the immune response of camels to infectious pathogens and as well as their response to vaccination.

\section{Animals}

\section{Materials and Methods}

Blood was collected from four camels (Camelus dromedaries; males aged between 6 and 8 years) at Omran slaughterhouse, Al Ahsaa, Eastern Province, Saudi Arabia.

The camels included in the study were apparently healthy and had no vaccination history. Blood was obtained by venepuncture of the vena jugularis externa into vacutainer tubes containing EDTA (Becton Dickinson, Heidelberg, Germany).

Mononuclear cells and whole leukocytes Separation Separation of camel mononuclear cells (PBMC) was performed according to a method used for separating 
bovine blood cells with modification (Hussen et al., 2016). Blood was layered on Ficoll-Isopaque (SigmaAldrich, Germany) and centrifuged at $10^{\circ} \mathrm{C}$ for $30 \mathrm{~min}$ at $3000 \mathrm{rpm}$. The interphase containing PBMC was washed 3 times in PBS (2000, 1500 and $1000 \mathrm{rpm})$ and finally suspended in MIF buffer (PBS containing bovine serum albumin $(5 \mathrm{~g} / \mathrm{L})$ and $\left.\mathrm{NaN}_{3}(0.1 \mathrm{~g} / \mathrm{L})\right)$. For the separation of human PBMC, human blood was layered on Ficoll-Isopaque and centrifuged at $10^{\circ} \mathrm{C}$ for $30 \mathrm{~min}$ at $3000 \mathrm{rpm}$. The interphase containing PBMC was washed 3 times in PBS (2000, 1500 and $1000 \mathrm{rpm}$ ) and finally suspended in MIF buffer. Whole camel and human leukocytes were separated by hypotonic lysis of erythrocytes. Blood was suspended in distilled water for $20 \mathrm{sec}$ and double concentrated PBS was added to restore tonicity. This was repeated (usually twice) until complete erythrolysis. Separated cells were finally suspended in MIF buffer at 5 x 106 cells $/ \mathrm{ml}$. Cell purity of separated PBMC and leukocytes was assessed by flow cytometry according to their forward scatter (FCS) and sideward scatter (SSC) properties and always exceeded $90 \%$. The mean viability of separated cells, as determined by exclusion of propidium iodide (2 $\mu \mathrm{g} / \mathrm{ml}$, Calbiochem, Germany) ranged $92 \% \pm 4 \%$ (Fig. 1).

A
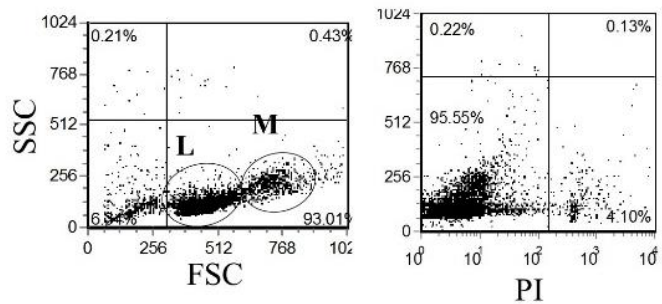

B
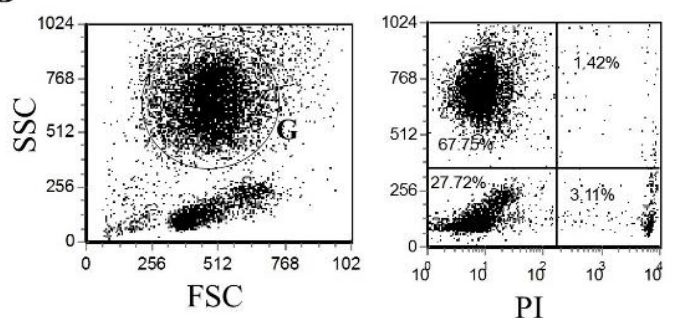

Fig. 1. Separation of camel blood PBMC and leukocytes and gating strategies. Camel PBMC were isolated by density gradient separation using Ficoll-Isopaque (A). Whole camel leukocytes were separated by hypotonic lysis of erythrocytes (B). Cell purity and viability of separated PBMC and leukocytes was assessed by flow cytometry according to their forward scatter (FCS) and sideward scatter (SSC) properties of the cells. The mean viability of separated cells was determined by exclusion of propidium iodide (PI). Gates were placed on granulocytes $(\mathrm{G})$, monocytes $(\mathrm{M})$ and lymphocytes (L) (as displayed in dot plot profile, side light scatter vs forward light scatter).

\section{Monoclonal antibodies}

The full list of mAbs (52 commercially available antibodies) used in this study are shown in Table 1.

Table 1. List of anti-human monoclonal antibodies.

\begin{tabular}{|c|c|c|c|}
\hline Antigen & Antibody clone & Source & Isotype \\
\hline $\mathrm{CD} 2$ & S5.2 & $\mathrm{BD}$ & $\mathrm{mIgG} 2 \mathrm{a}$ \\
\hline CD3 & SK7 (Leu-4) & $\mathrm{BD}$ & mIgG1 \\
\hline CD4 & SK3 & $\mathrm{BD}$ & mIgG1 \\
\hline CD5 & L17F12 & $\mathrm{BD}$ & $\mathrm{mIgG} 2 \mathrm{a}$ \\
\hline CD7 & M-T701 & $\mathrm{BD}$ & mIgG1 \\
\hline CD7 & $4 \mathrm{H} 9$ & $\mathrm{BD}$ & $\mathrm{mIgG} 2 \mathrm{a}$ \\
\hline CD8 & SK1 & $\mathrm{BD}$ & mIgG1 \\
\hline CD9 & M-L13 & $\mathrm{BD}$ & mIgG1 \\
\hline CD10 & HI10a & $\mathrm{BD}$ & mIgG1 \\
\hline CD11a & G43-25B & $\mathrm{BD}$ & $\mathrm{mIgG} 2 \mathrm{a}$ \\
\hline CD11b & ICRF44 & $\mathrm{BD}$ & mIgG1 \\
\hline $\mathrm{CD} 11 \mathrm{c}$ & S-HCL-3 & $\mathrm{BD}$ & $\mathrm{mIgG} 2 \mathrm{~b}$ \\
\hline CD11c & KB90 & Dako & mIgG1 \\
\hline CD13 & L138 & $\mathrm{BD}$ & mIgG1 \\
\hline CD14 & M5E2 & $\mathrm{BD}$ & $\mathrm{mIgG} 2 \mathrm{a}$ \\
\hline CD14 & TÜK4 & Biorad & $\mathrm{mIgG} 2 \mathrm{a}$ \\
\hline CD14 & $\mathrm{M} \varphi \mathrm{P} 9$ & BD & $\mathrm{mIgG} 2 \mathrm{~b}$ \\
\hline CD15 & MMA & $\mathrm{BD}$ & mIgM \\
\hline CD16 & B73.1 & $\mathrm{BD}$ & mIgG1 \\
\hline CD16 & KD1 & $\mathrm{BD}$ & $\mathrm{mIgG} 2 \mathrm{a}$ \\
\hline CD18 & 42557 & $\mathrm{BD}$ & mIgG1 \\
\hline CD19 & $4 \mathrm{G} 7$ & $\mathrm{BD}$ & mIgG1 \\
\hline CD19 & $\mathrm{SJ} 25 \mathrm{C} 1$ & $\mathrm{BD}$ & mIgG1 \\
\hline $\mathrm{CD} 20$ & L27 & $\mathrm{BD}$ & mIgG1 \\
\hline CD20 & B9E9 & Coulter & $\mathrm{mIgG} 2 \mathrm{a}$ \\
\hline CD20 & $2 \mathrm{H} 7$ & Abcam & $\mathrm{mIgG} 2 \mathrm{~b}$ \\
\hline CD22 & S-HCL-1 & BD & mIgG2b \\
\hline CD33 & P67.6 & $\mathrm{BD}$ & mIgG1 \\
\hline CD38 & HB7 & $\mathrm{BD}$ & mIgG1 \\
\hline CD38 & HIT2 & $\mathrm{BD}$ & mIgG1 \\
\hline CD45 & 2D1 & $\mathrm{BD}$ & mIgG1 \\
\hline CD55 (DAF) & JS11KSC2.3 & Coulter & mIgG1 \\
\hline CD56 & NCAM16.2 & $\mathrm{BD}$ & mIgG2b \\
\hline CD58 (LFA-3) & $1 \mathrm{C} 3$ & $\mathrm{BD}$ & $\mathrm{mIgG} 2 \mathrm{a}$ \\
\hline CD62L & DREG-56 & $\mathrm{BD}$ & mIgG1 \\
\hline CD64 & 42379 & $\mathrm{BD}$ & mIgG1 \\
\hline CD79a & HM47 & Coulter & mIgG1 \\
\hline CD79b & CB3.1 & $\mathrm{BD}$ & mIgG1 \\
\hline CD95 & DX2 & Dako & mIgG1 \\
\hline CD99 & TÜ12 & $\mathrm{BD}$ & mIgG2a \\
\hline CD126 & M5 & $\mathrm{BD}$ & mIgG1 \\
\hline CD182 & $6 \mathrm{C} 6$ & $\mathrm{BD}$ & mIgG1 \\
\hline TCR- $\alpha \beta$ & WT31 & $\mathrm{BD}$ & mIgG1 \\
\hline TCR- $\gamma / \delta$ & $11 \mathrm{~F} 2$ & $\mathrm{BD}$ & mIgG1 \\
\hline $\operatorname{IgM}$ & G20-127 & $\mathrm{BD}$ & mIgG1 \\
\hline Ig-lambda chain & $1-155-2$ & $\mathrm{BD}$ & mIgG1 \\
\hline Ig-Kappa chain & TB28-2 & $\mathrm{BD}$ & mIgG1 \\
\hline MHC-II & L243 & $\mathrm{BD}$ & $\mathrm{mIgG} 2 \mathrm{a}$ \\
\hline MHC-II & G46.2.6 & $\mathrm{BD}$ & mIgG1 \\
\hline bcl-2 & $\mathrm{Bcl}-2 / 100$ & $\mathrm{BD}$ & mIgG1 \\
\hline Kappa chain & TB28-2 & $\mathrm{BD}$ & mIgG1 \\
\hline Lambda chain & $1-155-2$ & $\mathrm{BD}$ & mIgG1 \\
\hline
\end{tabular}

Ig: Immunoglobulin; m: mouse; MHC-II: Major Histocompatibility Complex class II. 


\section{Immunofluorescence and flow cytometry}

Camel or human cells (PBMC or leucocytes; $4 \times 10^{5}$ ) were incubated with mAbs specific for human $C D$ antigens (Table 1) in PBS containing bovine serum albumin $(5 \mathrm{~g} / \mathrm{l})$ and $\mathrm{NaN}_{3}(0.1 \mathrm{~g} / \mathrm{l})$. After 30 minutes incubation $\left(4^{\circ} \mathrm{C}\right)$, cells were washed twice and analyzed on the flow cytometer. A Becton Dickinson FACSCalibur equipped with Cell Quest software (FACSCalibur (Becton Dickinson Biosciences, San Jose, California, USA) was used to collect the data. At least 100000 cells were collected and analyzed with the FCS Express software Version 3 (De Novo Software, Thornton, Ontario).

In order to exclude signals due to non-specific binding of mouse antibodies, negative isotype controls for mouse IgG1, IgG2a, IgG2b (from BD) and IgM (from Beckmann Coulter) were also included as part of the study.

\section{Results}

Flow cytometry was used to determine the reactivity of camel leukocytes with commercially available mAbs to human leukocyte markers. PBMC were isolated by density gradient centrifugation using Ficoll-Isopaque as standard method for studying the phenotype of PBMC without interfering effects of granulocytes (Fig. 1A). Whole leukocytes were separated by hypotonic lysis of erythrocytes (Fig. 1B). Separated camel or human cells were incubated with mouse mAbs specific for human leukocyte antigens or with mouse isotype control antibodies (Table 1) and were analyzed by flow cytometry. For the analysis of population-specific pattern of expression, gates were done for lymphocytes (L), monocytes (M) and granulocytes (G) (Fig.1). The profiles obtained for each mAb were then compared to the labeling pattern of reactivity obtained with human leukocytes.

\section{Reactivity of mAbs to human leukocyte antigens with} camel PBMC

The mAb 555923 (clone 6.7; from BD) is a FITClabelled mouse antibody against human CD18. The expression pattern of CD18 on human and camel PBMC is shown in Fig.2. The mAb 555923 stained all human and camel PBMC, although the expression on lymphocytes was comparatively lower than monocytes. Also for both species, a minor subpopulation of lymphocytes remained negative for mAb 555923.

The mAb 555380 (clone G43-25B; from BD) is a PElabelled mouse antibody directed against human CD11a. The expression pattern of CD11a on human and camel PBMC is shown in Fig.3. Although it stained all population of human leucocytes widely, mAb 555380 indicated a higher expression of CD11a on human monocytes and a subset of human lymphocytes. For camel cells, the mAb 555380 stained all cell populations weekly than human cells.
(A)

PBMC

(B)
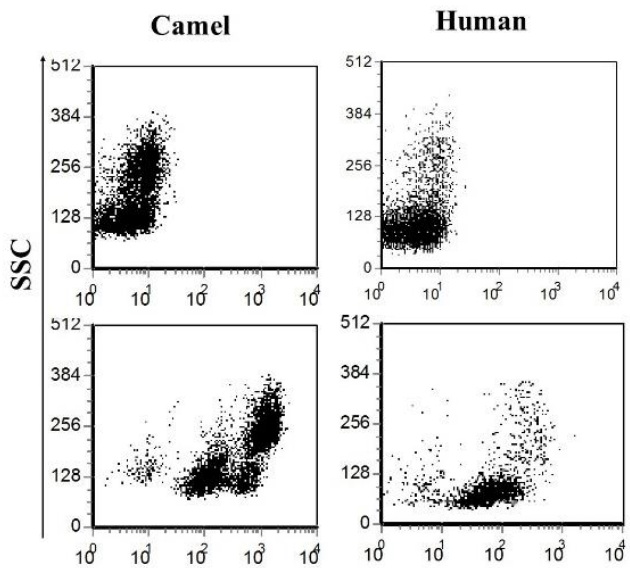

(C)

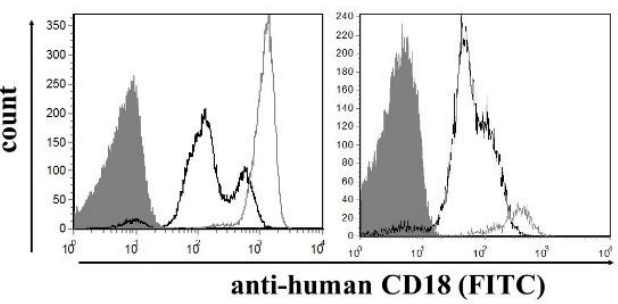

Fig. 2. Analysis of the expression pattern of CD18 on camel and human PBMC. Ficoll-separated camel or human PBMC were incubated with FITC-labelled mouse isotype control antibody (A) or the FITC-labelled monoclonal antibody 555923 (clone 6.7) specific for human CD18 (B) and analysed on the flow cytometer. The expression pattern of CD18 on camel or human PBMC was analysed by plotting SSC against CD18 expression. After gating lymphocytes and monocytes according to their forward and side scatter characteristics, the expression density of CD18 was shown in an overlapping histogram (C).

However a higher expression could be seen for camel monocytes and a subset of lymphocytes.

The mAb 557743 (clone ICRF44; from BD) is a PECy7-labelled mouse antibody directed against human CD11b. The expression pattern of CD11b on human and camel PBMC is shown in Fig.4. For both human and camel PBMC the mAb 557743 stained only monocytes and a minor subpopulation of lymphocytes, whereas the majority of lymphocytes was negative for this antibody.

The mAbs, 555398 (clone M5E2; from BD) and MCA1568PE (clone Tük4; from Bio-Rad) are PElabelled mouse antibodies directed against human CD14. Both mAbs showed a similar staining pattern for human and camel PBMC. In both species, only the monocytes population was stained positively with both CD14 antibodies. The expression pattern of CD14 (data shown only for the clone M5E2) on human and camel PBMC is shown in Fig.5. The mouse mAb 347403 (clone L243; from BD) is an APC-labelled antibody directed against human HLA-DR antigen. 


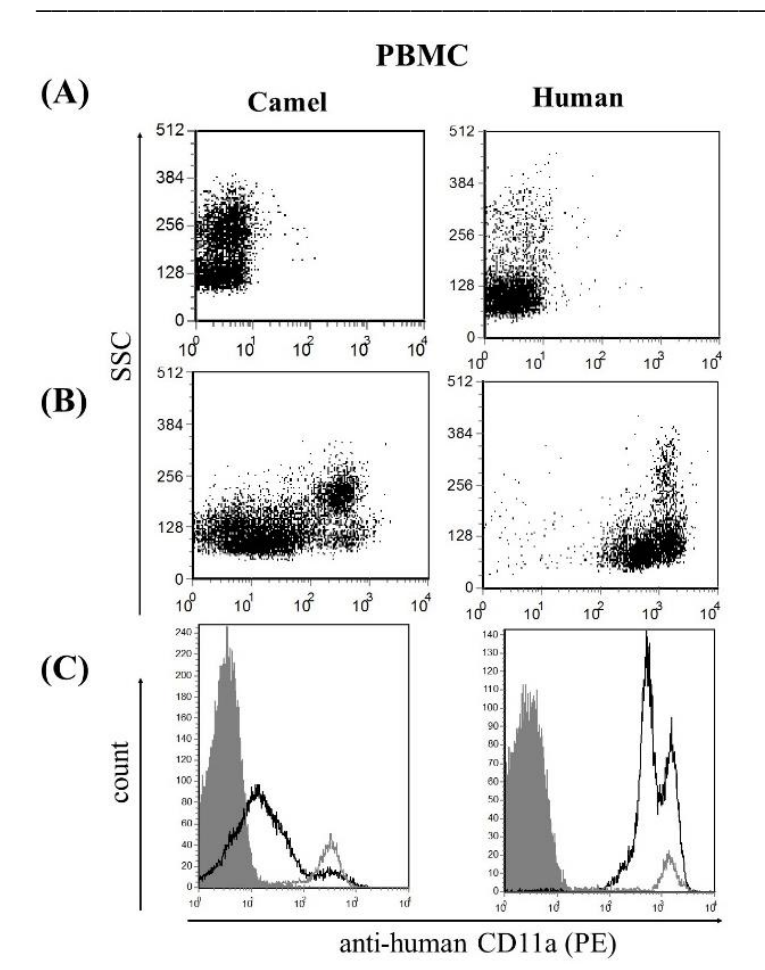

Fig. 3. Analysis of the expression pattern of CD11a on camel and human PBMC. Ficoll-separated camel or human PBMC were incubated with PE-labelled with mouse isotype control antibody (A) or the PE-labelled monoclonal antibody 555380 (clone G43-25B) specific for human CD11a (B) and analysed on the flow cytometer. The expression pattern of CD11a on camel or human PBMC was analysed by plotting SSC against CD11a expression. After gating lymphocytes and monocytes according to their forward and side scatter characteristics, the expression density of CD11a was shown in an overlapping histogram (C).

For human PBMC the mAb 347403 stained only the monocytes population and a subpopulation of lymphocytes, which is expected to be B cells. For camel cells however, monocytes showed only week reactivity with this mAb. Only a minor subset of camel lymphocytes stained weekly positive with this $\mathrm{mAb}$.

Reactivity of mAbs to human leukocyte antigens with camel granulocytes

Camel and human leukocytes were separated by hypotonic lysis of erythrocytes and were labelled with $\mathrm{mAb}$ to human $\mathrm{CD}$ antigens. Camel granulocytes showed reactivity only to three mAbs to human CD18, CD11a and CD11b (data not shown). The mAbs 555923 (FITC-labelled mouse anti-human CD18) and 555380 (PE-labelled mouse anti human CD11a) stained both human and camel granulocytes widely positive. However, a minor subpopulation of human granulocytes remained negative for the mAb 555923. Although the anti-human CD11b mAb 557743 stained both human and camel granulocytes positively, the reactivity of camel granulocytes was more weekly than that of human cells.

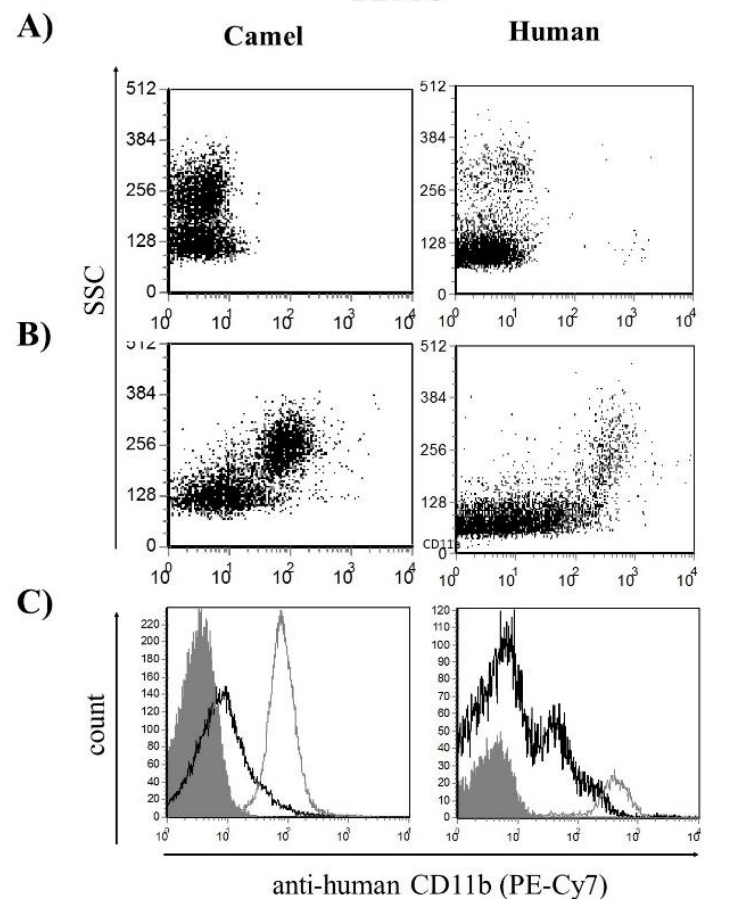

Fig. 4. Analysis of the expression pattern of CD11b on camel and human PBMC. Ficoll-separated camel or human PBMC were incubated with PE-Cy7-labelled mouse isotype control antibody (A) or the PE-Cy7-labelled monoclonal antibody 557743 (clone ICRF44) specific for human CD11b (B) and analysed on the flow cytometer. The expression pattern of CD11b on camel or human PBMC was analysed by plotting SSC against CD11b expression. After gating lymphocytes and monocytes according to their forward and side scatter characteristics, the expression density of CD11b was shown in an overlapping histogram $(\mathrm{C})$.

Only for human cells, a minor subpopulation of granulocytes remained negative for the mAb 557743.

\section{Discussion}

In comparison to the progress that has been made in the characterization of camel immunoglobulins (HamersCasterman et al., 1993; Muyldermans, 2013), few data are available on cellular immunity of camels in health or disease.

MAbs to leukocyte antigens have been considered as highly important tools for the analysis of cellular immunology in human (Maecker et al., 2012) as well as in different animal species (Hopkins et al., 1993; Lunn et al., 1998; Schafer and Burger, 2012; Duvel et al., 2014; Gerner et al., 2015). The lack of mAbs that define camel immune cells represents one of the major difficulties in studying the camel cellular immune response.

As the production of mAbs is very costly, the objective of the present study was to screen existing mAbs to human leukocyte antigens, immunoglobulin (Ig) chains and major MHC for their reactivity with camel leukocytes. 
A)

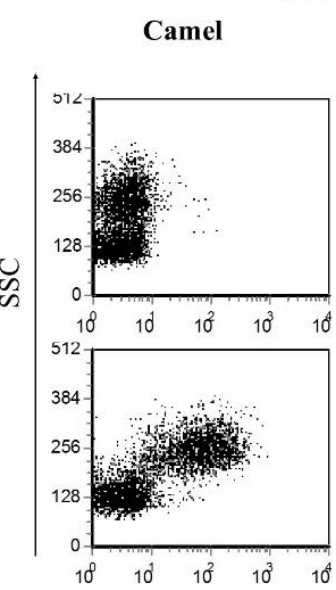

C)

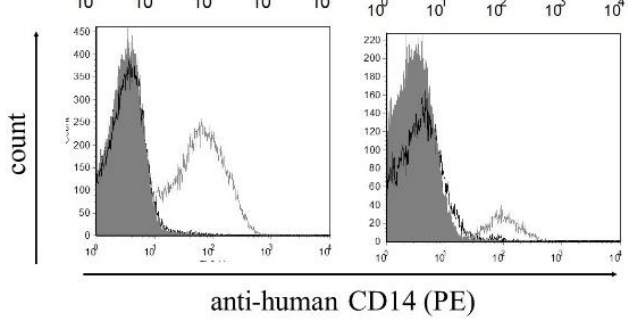

Fig. 5. Analysis of the expression pattern of CD14 on camel and human PBMC. Ficoll-separated camel or human PBMC were incubated with PE-labelled mouse isotype control antibody (A) or with the PE-labelled monoclonal antibody 555398 (clone M5E2) specific for human CD14 (B) and analysed on the flow cytometer. The expression pattern of CD14 on camel or human PBMC was analysed by plotting SSC against CD14 expression. After gating on lymphocytes or monocytes according to their forward and side scatter characteristics, the expression density of $\mathrm{CD} 14$ was shown in an overlapping histogram (C).

Two-parameter flow cytometry has been proven as a useful tool to study the cross-reactivity of mAbs developed against leukocyte differentiation antigens of one species with leukocyte antigens of other species (MacHugh et al., 1991; Naessens et al., 1993; Maecker et al., 2012). The specificity of a mAbs to a given antigen can be predicted according to the flowcytometric pattern of expression of that molecule on labeled leukocytes (MacHugh et al., 1991; Naessens et al., 1993; Davis et al., 2007; Davis and Hamilton, 2008).

$\mathrm{CD} 18$ is the common $\beta_{2}$-chain ( $\beta_{2}$-integrin) for all three forms of CD11 (a, b, c) (Harris et al., 2000). It has been shown that CD18 is expressed on all human leukocytes (Drbal et al., 2000) which is in accordance with our results of staining human PBMC and granulocytes with the mouse mAb 555923 (clone 6.7). Similar to their human counterparts, camel PBMC and granulocytes showed expressed CD18. As the patterns of reactivity for camel and human PBMC and granulocytes were identical so the homology of the proteins stained may be assumed.
Human CD11a is expressed together with CD18 as a hetero-dimer termed as leukocyte function antigen 1 (LFA-1). LFA-1 is the most important integrin expressed by all human leukocytes that regulate cell migration through binding to ICAM-1,-2 or-3 (van Kooyk and Figdor, 2000). As camel PBMC and granulocytes stained weekly than their human counterparts with the mAb 555380 (clone G43-25B) specific for human CD11a, it is likely that this mAb has a lower affinity for camel CD11a. However, the staining pattern of cell populations in both species with a higher staining density for human and camel granulocytes, monocytes and a subset of lymphocytes indicates that this antibody detects CD11a in camels. $\mathrm{CD} 11 \mathrm{~b}$ is expressed as a hetero-dimer with CD18 (also termed Mac-1 or CR3) mainly on myeloid cells (granulocytes and monocytic cells) (Imhof and Aurrand-Lions, 2004; Nicholson et al., 2007) with a lower expression on lymphocytes like NK cells, $\gamma \delta \mathrm{T}$ cells and a small subset of CD8+ T cells (Fiorentini et al., 2001; Graff and Jutila, 2007). The higher staining intensity of human and camel granulocytes and monocytes with the mAb 557743 (clone ICRF44) specific for human $\mathrm{CD} 11 \mathrm{~b}$ and the weaker staining of lymphocytes indicates that this $\mathrm{mAb}$ recognizes $\mathrm{CD} 11 \mathrm{~b}$ in both species.

CD14 is a co-receptor for bacterial lipopolysaccharides and is mainly expressed on blood monocytes (Hussen et al., 2013). Three clones of antibodies specific to human CD14 have been tested for cross-reactivity with camel leukocytes. Only the two mAbs 555398 (clone M5E2) and MCA1568PE (clone Tük4) stained camel monocytes but not granulocytes or lymphocytes, which indicates that these mAbs recognize camel CD14.

Human MHC class II antigen (HLA-DR) is constitutively expressed on professional antigenpresenting cells like dendritic cells, B cells, and monocytes (Abeles et al., 2012). The mAb 347403 (clone L243), which stained human monocytes and a population of lymphocytes, induced only a weak staining response in camel monocytes and lymphocytes. It is likely that this mAb has a low affinity to camel MHC-II molecules.

In summary, the present study aimed at providing the field of immunology with new antibodies to camel leukocyte antigens. Although some useful mAbs (about $10 \%$ of studied $52 \mathrm{mAb}$ ) have been identified in the present study, which may contribute to fill the gap of available reagents for studying the immune response of camels to infectious pathogens and their response to vaccination, there is a clear need for developing mAbs to more camel leukocyte antigens. Also further crossreactivity studies using mAbs against leukocyte antigens of animals with close sequence homology to camels like cattle or pigs could be helpful in identifying more cross-reactive antibodies. 


\section{Acknowledgements}

The cooperation and assistance of the Al Omran slaughter house personal is greatly acknowledged Conflict of interest

The authors declare that there is no conflict of interest.

\section{References}

Abeles, R.D., McPhail, M.J., Sowter, D., Antoniades, C.G., Vergis, N., Vijay, G.K., Xystrakis, E., Khamri, W., Shawcross, D.L., Ma, Y., Wendon, J.A. and Vergani, D. 2012. CD14, CD16 and HLADR reliably identifies human monocytes and their subsets in the context of pathologically reduced HLA-DR expression by CD14(hi) /CD16(neg) monocytes: Expansion of CD14(hi) /CD16(pos) and contraction of CD14(lo) /CD16(pos) monocytes in acute liver failure. Cytometry A 81(10), 823-834.

Al-Mohammed Salem, K., Badi, F.A., Al Haroon, A.I. and Alluwaimi, A.M. 2012. The Cellular Populations of Normal Camel (Camelus dromedaries) Milk. Open J. Vet. Med. 2, 262-265.

Davis, W.C., Davis, J.E. and Hamilton, M.J. 1995. Use of monoclonal antibodies and flow cytometry to cluster and analyze leukocyte differentiation molecules. Methods Mol. Biol. 45, 149-167.

Davis, W.C., Drbal, K., Mosaad, A.E., Elbagory, A.R., Tibary, A., Barrington, G.M., Park, Y.H. and Hamilton, M.J. 2007. Use of flow cytometry to identify monoclonal antibodies that recognize conserved epitopes on orthologous leukocyte differentiation antigens in goats, llamas, and rabbits. Vet. Immunol. Immunopathol. 119(1-2), 123-130.

Davis, W.C. and Hamilton, M.J. 2008. Use of flow cytometry to develop and characterize a set of monoclonal antibodies specific for rabbit leukocyte differentiation molecules. J. Vet. Sci. 9(1), 51-66.

Drbal, K., Angelisova, P., Cerny, J., Pavlistova, D., Cebecauer, M., Novak, P. and Horejsi, V. 2000. Human leukocytes contain a large pool of free forms of CD18. Biochem. Biophys. Res. Commun. 275(2), 295-299.

Duvel, A., Maass, J., Heppelmann, M., Hussen, J., Koy, M., Piechotta, M., Sandra, O., Smith, D.G., Sheldon, I.M., Dieuzy-Labaye, I., Zieger, P. and Schuberth, H.J. 2014. Peripheral blood leukocytes of cows with subclinical endometritis show an altered cellular composition and gene expression. Theriogenology 81(7), 906-917.

Fiorentini, S., Licenziati, S., Alessandri, G., Castelli, F., Caligaris, S., Bonafede, M., Grassi, M., Garrafa, E., Balsari, A., Turano, A. and Caruso, A. 2001. CD11b expression identifies CD8+CD28+ T lymphocytes with phenotype and function of both naive/memory and effector cells. J. Immunol. 166(2), 900-907.
Gerner, W., Talker, S.C., Koinig, H.C., Sedlak, C., Mair, K.H. and Saalmuller, A. 2015. Phenotypic and functional differentiation of porcine alphabeta T cells: current knowledge and available tools. Mol. Immunol. 66(1), 3-13.

Graff, J.C. and Jutila, M.A. 2007. Differential regulation of $\mathrm{CD} 11 \mathrm{~b}$ on gammadelta $\mathrm{T}$ cells and monocytes in response to unripe apple polyphenols. J. Leukoc. Biol. 82(3), 603-607.

Hamers-Casterman, C., Atarhouch, T., Muyldermans, S., Robinson, G., Hamers, C., Songa, E.B., Bendahman, N. and Hamers, R. 1993. Naturally occurring antibodies devoid of light chains. Nature 363(6428), 446-448.

Harris, E.S., McIntyre, T.M., Prescott, S.M. and Zimmerman, G.A. 2000. The leukocyte integrins. J. Biol. Chem. 275(31), 23409-23412.

Hopkins, J., Ross, A. and Dutia, B.M. 1993. Summary of workshop findings of leukocyte antigens in sheep. Vet. Immunol. Immunopathol. 39, 49-59.

Hussen, J., Duvel, A., Sandra, O., Smith, D., Sheldon, I.M., Zieger, P. and Schuberth, H.J. 2013. Phenotypic and functional heterogeneity of bovine blood monocytes. PLoS One 8(8), e71502.

Hussen, J., Koy, M., Petzl, W. and Schuberth, H.J. 2016. Neutrophil degranulation differentially modulates phenotype and function of bovine monocyte subsets. Innate Immun. 22(2), 124-137.

Imhof, B.A. and Aurrand-Lions, M. 2004. Adhesion mechanisms regulating the migration of monocytes. Nat. Rev. Immunol. 4(6), 432-444.

Lunn, D.P., Holmes, M.A., Antczak, D.F., Agerwal, N., Baker, J., Bendali-Ahcene, S., Blanchard-Channell, M., Byrne, K.M., Cannizzo, K., Davis, W., Hamilton, M.J., Hannant, D., Kondo, T., Kydd, J.H., Monier, M.C., Moore, P.F., O'Neil, T., Schram, B.R., Sheoran, A., Stott, J.L., Sugiura, T. and Vagnoni, K.E. 1998. Report of the Second Equine Leucocyte Antigen Workshop, Squaw valley, California, July 1995. Vet. Immunol. Immunopathol. 62(2), 101-143.

MacHugh, N.D., Bensaid, A., Howard, C.J., Davis, W.C. and Morrison, W.I. 1991. Analysis of the reactivity of anti-bovine CD8 monoclonal antibodies with cloned $\mathrm{T}$ cell lines and mouse Lcells transfected with bovine CD8. Vet. Immunol. Immunopathol. 27(1-3), 169-172.

Maecker, H.T., McCoy, J.P. and Nussenblatt, R. 2012. Standardizing immunophenotyping for the Human Immunology Project. Nat. Rev. Immunol. 12(3), 191-200.

Muyldermans, S. 2013. Nanobodies: natural singledomain antibodies. Annu. Rev. Biochem. 82, 775797.

Naessens, J., Olubayo, R.O., Davis, W.C. and Hopkins, J. 1993. Cross-reactivity of workshop antibodies 
with cells from domestic and wild ruminants. Vet. Immunol. Immunopathol. 39(1-3), 283-290.

Nicholson, G.C., Tennant, R.C., Carpenter, D.C., Sarau, H.M., Kon, O.M., Barnes, P.J., Salmon, M., Vessey, R.S., Tal-Singer, R. and Hansel, T.T. 2007. A novel flow cytometric assay of human whole blood neutrophil and monocyte CD11b levels: upregulation by chemokines is related to receptor expression, comparison with neutrophil shape change, and effects of a chemokine receptor (CXCR2) antagonist. Pulm. Pharmacol. Ther. 20(1), 52-59.

Schafer, H. and Burger, R. 2012. Tools for cellular immunology and vaccine research the in the guinea pig: monoclonal antibodies to cell surface antigens and cell lines. Vaccine 30(40), 5804-5811.

van Kooyk, Y. and Figdor, C.G. 2000. Avidity regulation of integrins: the driving force in leukocyte adhesion. Curr. Opin. Cell Biol. 12(5), 542-547.

Zidan, M., Kassem, A. and Pabst, R. 2000a. Megakaryocytes and platelets in the spleen of the dromedary camel (Camelus dromedarius). Anat. Histol. Embryol. 29(4), 221-224.

Zidan, M., Schuberth, H.J. and Pabst, R. 2000b. Immunohistology of the splenic compartments of the one humped camel (Camelus dromedarius). Vet. Immunol. Immunopathol. 74(1-2), 17-29. 